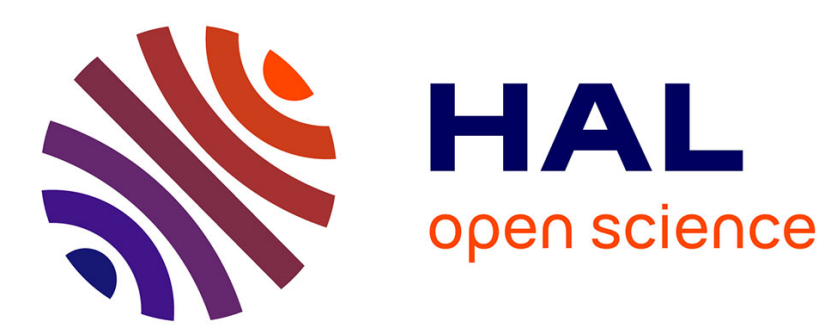

\title{
Intersensorialité humaine et cognition sociale
} Joël Candau

\section{- To cite this version:}

Joël Candau. Intersensorialité humaine et cognition sociale. Communications, 2010, 86 (1), pp.25 36. $10.3917 /$ commu.086.0025 . halshs-01756537

\section{HAL Id: halshs-01756537 https://shs.hal.science/halshs-01756537}

Submitted on 6 Feb 2020

HAL is a multi-disciplinary open access archive for the deposit and dissemination of scientific research documents, whether they are published or not. The documents may come from teaching and research institutions in France or abroad, or from public or private research centers.
L'archive ouverte pluridisciplinaire HAL, est destinée au dépôt et à la diffusion de documents scientifiques de niveau recherche, publiés ou non, émanant des établissements d'enseignement et de recherche français ou étrangers, des laboratoires publics ou privés. 


\title{
INTERSENSORIALITÉ HUMAINE ET COGNITION SOCIALE Joël Candau
}

\author{
Le Seuil | «Communications»
}

$2010 / 1 n^{\circ} 86 \mid$ pages 25 à 36

ISSN 0588-8018

ISBN 9782021022551

Article disponible en ligne à l'adresse :

https://www.cairn.info/revue-communications-2010-1-page-25.htm

\section{Pour citer cet article :}

Joël Candau, «Intersensorialité humaine et cognition sociale », Communications 2010/1 ( $\left.\mathrm{n}^{\circ} 86\right)$, p. 25-36.

DOI 10.3917/commu.086.0025

Distribution électronique Cairn.info pour Le Seuil.

(C) Le Seuil. Tous droits réservés pour tous pays.

La reproduction ou représentation de cet article, notamment par photocopie, n'est autorisée que dans les limites des conditions générales d'utilisation du site ou, le cas échéant, des conditions générales de la licence souscrite par votre établissement. Toute autre reproduction ou représentation, en tout ou partie, sous quelque forme et de quelque manière que ce soit, est interdite sauf accord préalable et écrit de l'éditeur, en dehors des cas prévus par la législation en vigueur en France. Il est précisé que son stockage dans une base de données est également interdit. 


\section{Intersensorialité humaine et cognition sociale}

\section{Introduction.}

Traditionnellement, l'influence des thèses condillaciennes a conduit à modéliser la communication entre un sujet et le monde qui l'entoure à l'intérieur de strictes frontières sensorielles : il y aurait la communication olfactive, la communication tactile, la communication visuelle, etc. Cette inclination à penser des sens séparés est également manifeste dans leurs représentations hiérarchisées ${ }^{1}$. Il n'est pas certain, cependant, que cette manière de voir corresponde à l'ontologie profonde de nos expériences sensibles. En effet, des données anatomophysiologiques et ethnographiques récentes plaident en faveur d'une autre hypothèse, celle du traitement multisensoriel - ou, plus exactement, intersensoriel - des stimuli.

Dans la première partie de l'article, j'étaye cette hypothèse en rappelant les mécanismes physiologiques sous-jacents à l'expérience intersensorielle. Dans la seconde partie, j'illustre l'hypothèse avec trois exemples. Le premier est un ensemble de données sur le langage naturel des odeurs recueillies en situation expérimentale par le groupe de recherche MOD ("Molécules, olfaction, discours») de l'Université de Nice-Sophia Antipolis. Le deuxième relève de mes enquêtes ethnographiques auprès de professionnels confrontés à des environnements olfactifs sévères, notamment les fossoyeurs et les thanatopracteurs. Le troisième exemple est plus personnel et, par ce fait même, ne prétend pas à la rigueur de l'observation scientifique. Il s'agit du compte rendu de ma participation à un repas «en aveugle ", intitulé La cena de casi todos los sentidos ${ }^{2}$. Pour chacun de ces trois cas, je soutiens que le traitement de l'information non seulement n'a jamais été unisensoriel mais, en outre, a pu déjouer un certain nombre d'attentes en matière de hiérarchie et de séquences sensorielles. En conclusion, j'en souligne les implications anthropologiques dans le domaine de la cognition sociale. 


\section{Les bases biologiques de l'intersensorialité.}

Le corps est un support mémoriel performant, à plusieurs titres. Il porte les multiples traces de ce que nous avons vécu, sa dégradation continue, sans rémission possible, nous rappelle la longueur des distances traversées, et la façon dont il nous agit - postures, gestuelles et, plus généralement, tous les encodages protomémoriels ${ }^{3}$ - contribue à raviver notre mémoire autobiographique $^{4}$. Le corps, cependant, n'est pas seulement mémoire du passé. Il est également mémoire au présent et au futur immédiat. Son architecture, fruit de notre histoire évolutive, oriente nos manières de penser ${ }^{5}$. Par exemple, les trois grandes oppositions qui structurent notre cognition spatiale - haut/bas, devant/derrière, gauche/droite - correspondent à notre perception de la position des différentes parties de notre corps dans l'espace $^{6}$ : tête/pieds, face-buste-ventre/nuque-dos-fesses, symétrie des membres inférieurs et supérieurs, cela en relation avec les faits objectifs de notre verticalité, d'une morphologie dorso-ventrale et de la latéralisation manuelle. À des degrés variables ${ }^{7}$ et selon des formes culturelles diverses, cette perception semble partagée par tous les êtres humains.

S'il oriente nos manières de penser, le corps oriente aussi, indissociablement, nos manières d'agir et, notamment, d'interagir avec les autres. Tant par ses productions que par ses réceptions sensorielles, il est, au quotidien, un média de l'information intersubjective ou, si l'on veut, un sociotransmetteur ${ }^{8}$, c'est-à-dire une entité du monde qui, métaphoriquement, remplit entre individus la même fonction que les neurotransmetteurs entre neurones : favoriser les connexions.

Selon quelles modalités ces messages sensoriels nous connectent-ils aux mondes physique et social? Des travaux les plus récents en sciences et neurosciences cognitives se dégage un intérêt croissant pour l'intersensorialité. On désigne ainsi les multiples processus d'interaction et d'intégration entre les différents sens (interactions intermodales) qui débordent les processus similaires internes à un sens particulier (interactions intramodales consistant, par exemple, à unifier en une seule scène mentale la forme, la matière et la couleur d'un objet lors de sa perception visuelle). Grâce aux techniques d'imagerie cérébrale, on a pu identifier de multiples zones de convergence des différents messages sensoriels dans le cerveau ${ }^{9}$. Non seulement les sensations relatives à un sens spécifique peuvent être affectées par les interactions avec d'autres sens, mais les jugements eux-mêmes (les perceptions) en subissent les effets.

Le premier exemple auquel on peut songer, parce qu'il a fait l'objet d'une littérature considérable ${ }^{10}$, est celui de la synesthésie, classiquement définie comme une expérience physique de croisement des signaux senso- 
riels telle que la stimulation d'un sens en éveille d'autres. On cite toujours Kandinsky, qui voyait «ses » couleurs en écoutant Wagner, ou Nabokov, qui, comme sa mère, faisait correspondre à la lettre $b$ la couleur terre de Sienne brûlée et à la lettre $t$ le vert pistache. En fait, il y a une très grande variété de cas de synesthésie, unidirectionnels ${ }^{11}$ ou bidirectionnels, tels que l'association d'un nombre à une couleur ${ }^{12}$, d'un mot à un goût ${ }^{13}$, ou encore le cas extraordinaire du sujet E.S., chez qui un intervalle musical induit une sensation gustative ${ }^{14}$.

Doit-on voir dans la synesthésie l'expression de l'aptitude humaine au traitement intermodal des messages sensoriels ou bien est-elle une faculté exceptionnelle partagée seulement par quelques individus? Nous naissons tous, probablement, avec une aptitude plus ou moins grande à la synesthésie, car les voies sensorielles sont mal définies chez le tout jeune enfant. C'est seulement pendant son ontogenèse que chaque sens s'autonomise, sauf chez les véritables synesthètes, qui continuent à inhiber le tri entre les différents messages sensoriels. Ces individus sont très peu nombreux et l'on sait aujourd'hui, grâce aux techniques d'imagerie cérébrale, que leur faculté a une base neuronale et génétique. Il serait donc erroné de voir dans la synesthésie la preuve d'une compétence intersensorielle de tous les êtres humains, même si tout un chacun, à l'aide de certaines drogues, peut atteindre des expériences synesthésiques, effet possible de la réactivation d'une disposition présente à la naissance.

L'existence d'une plasticité intermodale (cross-modal plasticity) de notre esprit-cerveau est un argument plus convaincant en faveur de l'hypothèse d'une prédisposition intersensorielle pan-humaine. Elle survient lorsqu'une aire du cortex spécifique à un sens se substitue à une autre aire sensorielle, généralement par compensation à la suite d'une lésion. Le cortex visuel primaire, par exemple, est activé quand des aveugles accomplissent des tâches tactiles telles que la lecture du braille ${ }^{15}$. Ce phénomène est bien connu, mais, en défense de ma thèse d'une intersensorialité massive de l'être humain, il est intéressant de considérer les deux hypothèses concurrentes avancées pour l'expliquer. La première est celle d'une réorganisation neuronale (the reorganization hypothesis) qui se manifeste sous la forme de l'apparition de nouvelles voies et de nouvelles connexions lorsqu'une région du cerveau est lésée. Selon la seconde hypothèse (the unmasking hypothesis), la lésion induit le dévoilement et le renforcement de connexions neuronales préexistantes. Or récemment ${ }^{16}$ ont été découverts des phénomènes de plasticité (la stimulation de l'aire visuelle chez des aveugles provoque la sensation du toucher sur la langue) apparus en moins d'une semaine, ce qui est un temps trop court pour que soient créées de nouvelles voies neuronales (the reorganization hypothesis). Ces données expérimentales plaident donc en faveur de l'hypothèse du dévoilement, 


\section{Joël Candau}

selon laquelle le phénomène de plasticité consiste en l'utilisation de voies d'intégration multisensorielle déjà existantes. Chez l'homme, l'interdépendance entre les différents sens, latente ou manifeste, serait alors la norme bien plus que l'exception.

On peut voir une autre expression de notre tropisme intersensoriel dans l'exigence de cohérence perceptuelle manifestée par les êtres humains lors de diverses expériences sensorielles: par exemple, un son élevé est plus compatible avec une lumière intense ${ }^{17}$, la perception d'un son peut influencer la perception visuelle ${ }^{18}$, la vue du mouvement des lèvres altère la réception auditive d'un phonème (effet McGurk ${ }^{19}$ ), il y a interaction entre la couleur d'un nom de couleur et son contenu sémantique (effet Stroop ${ }^{20}$ ), percevoir le mouvement des lèvres (vue) durant une conversation en situation de face-à-face active l'aire corticale auditive même en l'absence de sons ${ }^{21}$, etc.

Cette interdépendance est bien documentée entre différents registres sensoriels, par exemple entre la vue et le toucher. Chez les primates, il y a ainsi intégration des messages tactiles et visuels dans l'aire ventrale intrapariétale et dans le cortex prémoteur ventral ${ }^{22}$. Les savoir-faire haptiques intègrent largement des stimuli visuels (principalement), mais aussi d'autres stimuli sensoriels ${ }^{23}$. L'interdépendance est également attestée entre l'odorat et la vision. La dénomination d'un stimulus olfactif provoque l'activation d'une partie du cortex visuel primaire ${ }^{24}$. En situation expérimentale, les sujets associent préférentiellement certaines couleurs avec certaines odeurs (e.g. la couleur rose davantage avec l'odeur de fraise qu'avec celle de la menthe verte $\left.{ }^{25}\right)$. Ce lien entre perception des couleurs et des odeurs est également au cœur d'une expérience fameuse qui a montré que la description olfactive et gustative d'un vin blanc coloré artificiellement en rouge est sous l'emprise de cette coloration ${ }^{26}$. Selon d'autres travaux, les stimuli olfactifs peuvent moduler la perception tactile ${ }^{27}$, la préhension d'objets ${ }^{28}$ ou encore la nociception : l'exposition des nouveau-nés à l'odeur du lait maternel au moment de la prise de sang néonatale réduit significativement la durée de leurs pleurs après la piqûre ${ }^{29}$. On sait, enfin, que les traitements des informations par la voie orthonasale (pour les molécules odorantes, la voie «normale», qui passe par nos narines et l'épithélium olfactif) et par la voie rétronasale (par laquelle les molécules à l'origine des sensations gustatives passent de la cavité buccale jusqu'aux fosses nasales) sont étroitement liés. La flaveur de l'aliment elle-même fait intervenir bien d'autres modalités sensorielles que la gustation et l'olfaction: perception thermique, perception des volumes et des textures ${ }^{30}$ (stéréognosie buccale), audition, vision, la résultante permettant « de caractériser l'aliment dans sa totalité ${ }^{31} »$. Les souvenirs olfactifs sont eux aussi au centre de plusieurs sensations ${ }^{32}$. La mémoire enregistre en même temps que le sti- 
mulus tout son contexte sensoriel et émotionnel, phénomène bien connu sous le nom de «syndrome de Proust » ${ }^{33}$. La large intégration de ces informations dans le néocortex, en particulier dans le cortex orbitofrontal, qui est le siège des plus hautes fonctions cognitives, représente sans doute un avantage adaptatif ${ }^{34}$ et signe une caractéristique de notre espèce en regard des autres animaux, tels les rongeurs, capables d'excellentes performances olfactives mais dotés d'une intégration néocorticale minimale ${ }^{35}$.

Bref, de ce survol rapide de la littérature, on est enclin à conclure à la réalité d'une forte intersensorialité humaine, toujours à l'œuvre quel que soit le sens privilégié dans notre rapport au monde. L' «écologie fonctionnelle de notre $\operatorname{corps}^{36}$ », selon les termes de Gordon Shepherd, ne peut jamais être réduite à une seule explication. Elle est dépendante de multiples facteurs, ce qui revient à dire, comme l'avait noté Simmel, qu'il y a toujours "fusion » de nos impressions sensibles ${ }^{37}$ ou encore, en termes proustiens, que «tout peut se transposer ${ }^{38}$ ». Il y a là un mécanisme essentiel de la conscience du corps, qui est elle-même un aspect fondamental de la conscience de soi $^{39}$ et de notre conscience des autres ${ }^{40}$. C'est une conclusion finalement très banale puisque nous savons tous que c'est ainsi que nous construisons une scène mentale. Les diverses modalités sensorielles, rappelle Derek Denton, sont «liées entre elles, au sein de la conscience, en une unité perceptive globale ${ }^{41} »$. Dans la même veine, le neurobiologiste Bernard Roques définit la conscience comme «une synthèse consciente des sensations ${ }^{42}$ », renvoyant ainsi à l'expérience phénoménologique: "Quand j'évoque un souvenir de mer en Bretagne, je la vois, je la sens et je l'entends à la fois ${ }^{43}$. $\gg$ Au fond, cela suggère que l'unisensorialité nous reste étrangère. Seuls existent des représentations, des actes et un espace péri-personnel multisensoriels. Pour reprendre une formule éloquente de Lamarck, "c'est notre être en entier qui sent ${ }^{44}$ », ce que suggèrent les données expérimentales et ethnographiques sur l'expression en langue des stimuli olfactifs.

\section{L'expression en langue de l'intersensorialité.}

Instable et imprécis, le langage naturel des odeurs emprunte massivement des termes « tirés d'autres données de l'intuition sensible ${ }^{45}$ ». Chez les sujets d'une expérience de reconnaissance d'odeurs menée en 2005 au laboratoire d'analyse sensorielle de l'Université de Nice-Sophia Antipolis ${ }^{46}$, la description olfactive s'appuie effectivement sur les autres sens. Ainsi, une odeur peut être qualifiée d' «humide », «amère », "acidulée », "sucrée », «légère », «lourde », elle peut «piquer », faire penser «à quelque chose de plat», etc. Plusieurs descriptions mettent en correspondance une odeur 


\section{Joël Candau}

avec une couleur. L'isobutylamine, dit un sujet, a « un peu une odeur verte, quand même, enfin, de verdure ». Pour un autre participant à l'expérience, l'alpha-pinène fait penser à «du bleu». La coumarine, elle, fait songer «à la couleur verte ». Le para-hydroxy-phényl-butanone évoque chez un sujet «la couleur marron» et, chez un autre, «la couleur rose», peut-être parce que «c'est doux comme un bonbon». L'anthranilate de méthyle est mis en rapport avec «la couleur jaune», sans doute parce que c'est "une odeur citronnée». Le phénylacétate d'éthyle, enfin, est mis en correspondance avec la couleur verte, ce qui paraît logique puisque cette substance, selon le sujet, «sent la nature, le maquis, l'eucalyptus », «c'est une odeur d'herbe ».

Ces données expérimentales corroborent celles que j'ai recueillies lors de mes enquêtes ethnographiques sur les savoirs et savoir-faire olfactifs ${ }^{47}$. L’odeur du noyé, déclare un médecin légiste, est «lourde », " forte », « intenable, terrible, collante», elle «vient nous tartiner». "C'est une odeur sourde, basse, à ne pas regarder ${ }^{48}$, résume un fossoyeur. D'autres fossoyeurs, à propos de l'odeur d'un corps en état de putréfaction, disent qu'elle «accroche», «pique» et «rentre dans les cheveux». Elle est «collante », renchérit l'un d'entre eux, utilisant le même descripteur qu'un sapeur-pompier qui, à l'instar de ses collègues, la qualifie encore de "puissante, bloquante, écœurante, pointue, perçante». C'est une odeur « dérangeante », qui «fait mal » et «nous tombe dessus », précise un fossoyeur; on la « reçoit» et il faut donc s'habituer à la «prendre ». Elle «tient», y compris après que l'on a pris soin de changer de tenue, affirment plusieurs représentants de ces professions. L'un d'entre eux, à propos de l'« odeur de la souffrance » dégagée selon lui par les défunts dont l'agonie fut difficile, évoque le «bruit de l'odeur». Selon un thanatopracteur, cette odeur «âcre », «agressive », «acide», " grasse », « reste collée dans la bouche», on la «garde sur soi », et on doit ensuite la "porter», tel un fardeau. Elle est "prenante », elle "s'imprègne », elle "s'étale comme un chewing-gum », se «dépose dans les sinus», puis reste «ancrée au niveau du front», confirme, à Nice, un employé de la morgue municipale. En milieu hospitalier, la description des odeurs du corps humain évoque également le caractère intersensoriel de l'expérience olfactive. Une odeur d'infection intense, estime une infirmière, "imprègne » ses vêtements et son corps, impression qui rejoint celle d'un fossoyeur décrivant l'ouverture de certaines tombes: «on est pris à la gorge », dit-il, «l'odeur, vous l'avez sur les habits». Une autre infirmière a l'impression d' " avaler » les odeurs dégagées par de mauvaises plaies.

On le voit, l'expression en langue des stimuli olfactifs peut se faire selon des modalités diverses. Elle prend appui ici sur le goût, là sur la vue ou le toucher, ou encore sur tous ces sens à la fois, mais toujours elle dénote un traitement intermodal de l'information. Il est possible qu'il n'y ait là qu'une 
spécificité du langage naturel des odeurs, sans que le lexique renvoie à une intersensorialité réelle. Ici, cependant, l'expérience personnelle peut conforter la thèse de l'intermodalité massive, dans les limites inhérentes à toute subjectivité. Privé très momentanément de la vue, j'ai eu l'occasion d'être particulièrement attentif à ce mode de traitement intermodal lors d'un dîner intitulé La cena de casi todos los sentidos, organisé à Barcelone en juillet 2006 à l'intention des intervenants dans un congrès de sciences sensorielles.

Après que nous eûmes été accueillis dans une pièce où flottait une odeur d'encens, on nous a bandé les yeux puis une personne nous a guidés, en nous tenant par l'épaule, vers une autre pièce que j'ai découverte en tâtonnant : une chaise, une table, probablement une salle de restaurant. Dès mon arrivée, j'ai entendu une voix féminine chuchoter à mon oreille : "; Abre la boca! » J'ai obtempéré et quelqu'un a glissé une substance au goût de pâte d'olives entre mes lèvres. Puis on a mis un verre entre mes mains, j'ai entendu - et perçu aux vibrations du verre - un liquide le remplir, je l'ai senti, j'en ai bu une gorgée et j'ai cru deviner du champagne. Le repas ensuite a commencé. Pendant près de deux heures, tous mes sens autres que la vue n'ont cessé d'être sollicités. Le goût et l'odorat, bien sûr, quand nous ont été servis différents vins et mets que je n'ai jamais su identifier avec certitude. Le toucher également: celui des aliments et des boissons, l'exploration tactile de l'espace et des objets à ma disposition et, aussi, les sensations agréables provoquées par les légers attouchements des cheveux, des bras ou des mains que nous prodiguaient les personnes chargées du service. Enfin, l'ouie, au travers des informations auditives : murmures ou exclamations des convives, bruits du service, musique romantique sur laquelle, toujours les yeux bandés, nous avons été invités à danser.

Mais je ne veux pas prolonger à l'excès la description d'un événement qui peut paraître anecdotique et qui, pourtant, est riche d'enseignements pour l'anthropologue sensoriel : 1) en permanence, j'ai intégré les différents stimuli sensoriels dans une sensation globale de très grande euphorie ; 2) personne voyante privée momentanément de la vue, je n'ai cessé de prendre appui sur cette intégration multisensorielle, de manière bien plus prononcée que d'ordinaire, pour construire des images mentales de la scène à laquelle je participais ; 3) contrairement à mes attentes, j'ai été particulièrement attentif aux informations auditives qui, toujours, sont venues moduler mes perceptions gustatives et olfactives. Pour clore le compte rendu de cette expérience, on me pardonnera d'évoquer une de ses facettes les plus personnelles, qui m'a passablement intrigué et qui, à mes yeux, montre par défaut l'importance du traitement intersensoriel des stimuli. À un moment de l'exploration tactile de mon environnement, j'ai rencontré la main de la personne qui se trouvait à la même table que moi et dont j'ignorais jusquelà la présence. J'ai alors pris un réel plaisir à lui caresser les doigts, plaisir 


\section{Joël Candau}

que ne laissait pas prédire mon orientation sexuelle: à la fin du repas, quand on nous a retiré les bandeaux qui nous aveuglaient, j'ai constaté que mon commensal était un homme, par ailleurs bien plus âgé que moi. Cette expérience hédonique inattendue et consécutive à une modification de mon équilibre intersensoriel habituel montre, me semble-t-il, que dans ce domaine de l'intersensorialité le corps nous agit bien plus que nous ne l'agissons. Il ordonne, nous lui obéissons. Il se met en mouvement, nous le suivons. Il décide, nous l'approuvons et le laissons alors ouvrir l'ensemble de nos sphères cognitivo-émotionnelles, y compris les plus intimes.

\section{Conclusion.}

En guise de conclusion, je me risquerai à poser une question qui, plus que d'autres, est largement spéculative : notre perception est-elle encapsulée dans divers modules propres à chaque sens ${ }^{49}$, comme on suppose qu'il en existe pour la détection des visages ou la perception des espèces vivantes $^{50}$, ou bien obéit-elle à des processus qui débordent largement ce traitement modulaire? Au vu de l'intersensorialité massive que j'ai voulu illustrer dans ce texte, il semble bien que le langage des sens s'émancipe d'un traitement strictement modulaire. Cette aptitude à dépasser l'information transmise par un module tout en prenant appui sur elle doit certainement offrir de multiples avantages. Elle contribue à l'incorporation du contexte. Elle permet des inférences multiples sur les expériences sensibles et, en particulier, avec le support du langage, favorise le partage efficace de celles-ci ou, du moins, la croyance dans ce partage avec autrui. La construction intermodale de la scène mentale qui en résulte permet probablement d'atténuer ou de compenser les singularités, voire les aberrations perceptives qui peuvent être induites, chez un individu, par le caractère idiosyncrasique d'un sens particulier, cela en réduisant, en quelque sorte, le vécu expérientiel à un dénominateur commun - une représentation partagée - qui garantit une meilleure correspondance avec l'ontologie du monde physique. Le corps, dans cette perspective, peut être considéré comme le plus efficace des sociotransmetteurs : tant par ses productions que par ses réceptions sensorielles, il est, au quotidien, un média particulièrement performant de l'information intersubjective. Véhicule du langage des sens, il participe ainsi de nos manières partagées d'être au monde.

Joël CANDAU

joelcandau@gmail.com Université de Nice-Sofia Antipolis 


\section{Intersensorialité humaine et cognition sociale}

\section{NOTES}

1. Dias, 2004.

2. http://www.casitodoslosentidos.com/

3. Candau, 1998 , p. $12-14$.

4. Dijkstra et al., 2007, p. 139-149.

5. Pfeifer et Bongard, 2006.

6. Candau, 2008.

7. Avec, peut-être, des exceptions, bien que celles-ci soient controversées. L'étude de la langue des Tenejapan du Mexique (le tzetal) laisse supposer que ceux-ci ne disposent pas de la structure linguistique et conceptuelle nécessaire à la distinction entre la gauche et la droite: Jérôme Dokic, «L'espace de la perception et de l'imagination», in Bouveresse et Rosat (eds), 2003, p. 90.

8. J'ai proposé d'appeler « sociotransmetteurs» toutes les choses meublant le monde - les objets tangibles ou intangibles, les êtres animés (leurs comportements et leurs productions) - qui permettent d'établir une chaîne causale cognitive entre au moins deux esprits-cerveaux: Candau, $2004 \mathrm{a}$

9. Driver et Noesselt, 2008.

10. Cytowic, 1989.

11. La synesthésie est unidirectionnelle lorsque, par exemple, la couleur provoque une perception sonore mais pas l'inverse.

12. Sagiv et al., 2006.

13. Simner et Ward, 2006.

14. Beeli et al., 2005.

15. Sadato et al., 1996.

16. Kupers et al., 2006 ; Kadosh et Walsh, 2006.

17. Berthoz, 2003, p. 35.

18. McDonald et al., 2000.

19. McGurk et MacDonald, 1976.

20. Stroop, 1935.

21. Calvert et al., 2001.

22. Mountcastle, 2005.

23. Gross et Ghazanfar, 2006.

24. Qureshy et al., 2000.

25. Demattè et al., 2006a.

26. Morrot et al., 2001.

27. Demattè et al., 2006b.

28. Castiello et al., 2006.

29. Mellier et al., 1997.

30. Rolls, 2006.

31. M. Chiva, «Le mangeur et le mangé: la complexité d'une relation fondamentale», in Giachetti (ed.), 1996, p. 21.

32. Gottfried et al., 2004.

33. Candau, 2004b.

34. De Gelder et Bertelson, 2003.

35. Shepherd, 2006.

36. Shepherd, 2004.

37. Simmel, 1981, p. 226

38. Proust. 1987, p. 79.

39. Ehrsson et al., 2004; Ehrsson et al., 2005.

40. Li et al., 2007. Sur les effets inconscients des odeurs, voir aussi Holland et al., 2005.

41. Denton, 2005, p. 266.

42. Bernard P. Roques, «Le rêve, la drogue et la conscience », in Fussman (ed.), 2006, p. 116. 


\section{Joël Candau}

43. Tadié et Tadié, 1999, p. 137.

44. Lamarck, 1809, p. 252-253.

45. Cassirer, 1972, p. 150-151.

46. Baccino et al., 2009.

47. Candau, 2000.

48. Candau et Jeanjean, 2006.

49. Raftopoulos, 2001.

50. Sperber et Hirschfeld, 2004.

RÉFÉRENCES

Baccino, Th., Cabrol-Bass, D., Candau, J., Meyer, C., Scheer, T., Vulladume, M., et Wathelet, O., 2009, "Sharing an Olfactory Experience: The Impact of Oral Communication », Food Quality and Preference, doi:10.1016/j.foodqual.2009.11.001.

Beeli, G., Esslen, M., et Jäncke, L., 2005, "When Coloured Sounds Taste Sweet », Nature, vol. 434 , p. 38.

Berthoz, A., 2003, La Décision, Paris, Odile Jacob.

Bouveresse, J., et Rosat, J.-J. (eds), 2003, Philosophies de la perception. Phénoménologie, grammaire et sciences cognitives, Paris, Odile Jacob.

Calvert, G., Brammer, M., et Campbell, R., 2001, «Cortical Substrates of Seeing Speech : Still and Moving Faces », Neuroimage, 13 (6), p. S513.

Candau, J., 1998, Mémoire et identité, Paris, PUF.

- 2000, Mémoire et expériences olfactives. Anthropologie d'un savoir-faire sensoriel, Paris, PUF.

- 2004a, "Les processus de la mémoire partagée », Pour, nº 181, p. 118-123.

- 2004b, "L'odeur médiane du couvre-lit de tante Léonie », VOIR [barré], no 28-29, p. 72-87.

- 2008, «Entre bouasque et bronde, un ouvrage pionnier en cognition spatiale», in Mélanges à Annie-Hélène Dufour, Aix-en-Provence, Presses universitaires de Provence, p. 57-73.

Candau, J., et Jeanjean, A., 2006, «Des odeurs à ne pas regarder », Terrain, no 47, p. 51-68.

Cassirer, E., 1972, La Philosophie des formes symboliques, III. La Phénoménologie de la connaissance, Paris, Minuit, p. 150-151.

Castiello, U., Zucco, G., Parma, V., Ansuini, C., et Tirindelli, R., 2006, "Cross-Modal Interactions between Olfaction and Vision when Grasping », Chemical Senses, 31 (7), p. 665-671.

Cyтоwic, R.E., 1989, Synesthesia: A Union of the Senses, New York, Springer-Verlag.

De Gelder, B., et Bertelson, P., 2003, «Multisensory Integration, Perception and Ecological Validity », Trends in Cognitive Sciences, vol. 7, $\mathrm{n}^{\circ}$ 10, p. 460-467.

Demattè, M., Sanabria, D., et Spence, C., 2006a, "Cross-modal Association between Odours and Colours », Chemical Senses, 31 (6), p. 531-538.

Demattè, M., Sanabria, D., Sugarman, R., et Spence, C., 2006b, "Crossmodal Interactions between Olfaction and Touch », Chemical Senses, 31 (4), p. 291-300.

Denton, D., 2005, Les Émotions primordiales et l'Éveil de la conscience, Paris, Flammarion.

Dins, N., 2004, La Mesure des sens. Les anthropologues et le corps humain au XIXe siècle, Paris, Aubier.

Dijkstra, K., Kaschak, M.P., et Zwan, R.A., 2007, «Body Postures Facilitates Retrieval of Autobiographical Memories », Cognition, 102, p. 139-149.

Driver, J., et Noesselt, T., 2008, «Multisensory Interplay Reveals Crossmodal Influences on "Sensory-Specific" Brain Regions, Neural Responses, and Judgments», Neuron, 57, p. 11-23.

Ehrsson, H., Holmes, N., et Passingham, R., 2005, « Touching a Rubber Hand : Feeling of Body Ownership Is Associated with Activity in Multisensory Brain Areas », The Journal of Neuroscience, 25 (45), p. 10564-10573. 


\section{Intersensorialité humaine et cognition sociale}

Ehrsson, H., Spence, C., et Passingham, R., 2004, «That's My Hand! Activity in Premotor Cortex Reflects Feeling of Ownership of a Limb», Science, vol. 305, p. 875-877.

Fussman, G. (ed.), 2006, Croyance, raison et déraison, Paris, Odile Jacob / Collège de France.

GiachetTi, I. (ed.), 1996, Identités des mangeurs, images des aliments, CNERNA-CNRS, «Polytechnica».

GotTfried, J., et al., 2004, «Remembrance of Odors Past: Human Olfactory Cortex in CrossModal Recognition Memory », Neuron, vol. 42, nº 4, p. 687-695.

Gross, Ch. G., et Ghazanfar, A.A., 2006, "A Mostly Sure-Footed Account of the Hand », Science, vol. 312 , p. 1314.

Holland, R.W., Hendriks, M., et Aarts, H., 2005, «Smells like Clean Spirit. Nonconscious Effects of Scent on Cognition and Behavior ", Psychological Science, vol. 16, nº 9, p. 689-693.

Kadosh, R.C., et Walsh, V., 2006, «Cognitive Neuroscience: Rewired or Crosswired Brains? », Current Biology, vol. 16, no 22, p. R962-R963.

Kupers, R., Fumal, A, Maertens de Noordhout, A., Gjedde, A., Schoenen, J., et Ptito, M., 2006, "Transcranial Magnetic Stimulation of the Visual Cortex Induces Somatotopically Organized Qualia in Blind Subjects », PNAS, vol. 103, nº 35, p. 13256-13260.

Lamarck, J.-B., 1809, Philosophie zoologique, Paris, Dentu.

LI, W.. et al., 2007, "Subliminal Smells Can Guide Social Preferences », Psychological Science, vol. $18, \mathrm{n}^{\circ} 12$, p. 1044-1049.

McDonald, J.J., Teder-Sälejärvi, W.A., et Hillyard, S.A., 2000, «Involuntary Orienting to Sound Improves Visual Perception », Nature, vol. 407, p. 906-908.

McGurk, H., et MacDonald, J., 1976, «Hearing Lips and Seeing Voices », Nature, vol. 264, p. 746748 .

Mellier, D., Bezard, S., et Caston, J., 1997, «Études exploratoires des relations intersensorielles olfaction-douleur », Enfance, n 1, p. 98-111.

Morrot, G., Brochet, F., et Dubourdieu, D., 2001, «The Color of Odors », Brain and Language, 79, p. 309-320.

Mountcastle, V.B., 2005, "The Sensory Hand. Neural Mechanisms of Somatic Sensation», Cambridge (MA), Harvard University Press.

Pfeifer, R., et Bongard, J., 2006, How the Body Shapes the Way We Think: A New View of Intelligence, Cambridge (MA), The MIT Press.

Proust, M., 1987, À la recherche du temps perdu. La Prisonnière, Paris, Robert Laffont.

Qureshy, A., et al., 2000, «Functional Mapping of Human Brain in Olfactory Processing: A PET Study », Journal of Neurophysiology, 84, p. 1656-1666.

Raftopoulos, A., 2001, "Is Perception Informationally Encapsulated? The Issue of the Theoryladeness of Perception », Cognitive Science, 25, p. 423-451.

Rolls, E., 2006, «Brain Mechanisms Underlying Flavour and Appetite », Philosophical Transactions of the Royal Society B: Biological Sciences, vol. 361, n 1471, p. 1123-1136.

Sadato, N., Pascual-Leone, A., Grafman, J., Ibañez, V., Deiber, M.P., Dold, G., et Hallett, M., 1996, «Activation of the Primary Visual Cortex by Braille Reading in Blind Subjects », Nature, 380 , p. 526-528.

Sagiv, N., Simner, J., Collins, J., Butterworth, B., et WARD, J., 2006, "What Is the Relationship between Synaesthesia and Visuo-spatial Number Forms?», Cognition, 101, p. 114-128.

Shepherd, G., 2004, "The Human Sense of Smell : Are We Better than We Think? », PLoS Biology, vol. $2, \mathrm{n}^{\circ} 5$, p. $0572-0575$.

- 2006, "Smell Images and the Flavour System in the Human Brain », Nature, 444, p. 316-321.

Simmel, G., 1981, Sociologie et épistémologie, Paris, PUF.

Simner, J., et WARD, J., 2006, «The Taste of Words on the Tip of the Tongue », Nature, vol. 444. p. 438.

Sperber, D., et Hirschfeld, L., 2004, «The Cognitive Foundations of Cultural Stability and Diversity », Trends in Cognitive Sciences, vol. 8, n 1, p. 40-46.

STROOP, J.R., 1935, "Studies of Interference in Serial Verbal Reactions », Journal of Experimental Psychology, 18, p. 643-622.

Tadié, J.-Y., et Tadié, M., 1999, Le Sens de la mémoire, Paris, Gallimard. 


\section{Joël Candau}

\section{RÉSUMÉ}

Traditionnellement, l'influence des thèses condillaciennes a conduit à modéliser la communication entre un sujet et le monde qui l'entoure à l'intérieur de strictes frontières sensorielles : il y aurait la communication olfactive, la communication tactile, la communication visuelle, etc. Cependant, des données anatomophysiologiques et ethnographiques récentes plaident en faveur d'une autre hypothèse, celle d'un traitement intersensoriel des stimuli.

Dans la première partie de l'article, j'étaye cette hypothèse en rappelant les mécanismes physiologiques sous-jacents à l'expérience intersensorielle. Dans la seconde partie, j’illustre l'hypothèse avec des données expérimentales et ethnographiques. En conclusion, j’en souligne les implications anthropologiques dans le domaine de la cognition sociale.

\section{SUMMARY}

Traditionally, under the influence of the Condillac thesis, we model communication between an individual and his environment within strict sensorial boundaries : olfactory communication, tactile communication, visual communication, and so forth. However, recent anatomophysiological and ethnographic data suggest another hypothesis, that of an intersensorial processing of stimuli.

In the first part of my text, I support this hypothesis by recalling the physiological mechanisms underlying the intersensorial experience. In the second part, I illustrate the hypothesis with experimental and ethnographic data. To conclude, I underline their anthropological implications in the field of social cognition. 